\title{
Le rôle et la place du français dans le processus de modernisation de l'empire ottoman
}

\section{Hanife Güven}

\section{QpenEdition \\ Journals}

Édition électronique

URL : https://journals.openedition.org/dhfles/2566

DOI : $10.4000 /$ dhfles.2566

ISSN : 2221-4038

Éditeur

Société Internationale pour l'Histoire du Français Langue Étrangère ou Seconde

Édition imprimée

Date de publication : 1 décembre 2001

ISSN : 0992-7654

\section{Référence électronique}

Hanife Güven, «Le rôle et la place du français dans le processus de modernisation de l'empire ottoman », Documents pour l'histoire du français langue étrangère ou seconde [En ligne], 27 | 2001, mis en ligne le 31 janvier 2014, consulté le 27 mai 2021. URL : http://journals.openedition.org/dhfles/2566 ; DOI : https://doi.org/10.4000/dhfles.2566

Ce document a été généré automatiquement le 27 mai 2021.

(c) SIHFLES 


\title{
Le rôle et la place du français dans le processus de modernisation de l'empire ottoman
}

\author{
Hanife Güven
}

\section{Introduction}

1 Pour parler des premiers contacts franco-turcs il faudrait remonter jusqu'au Moyen Age quand les Occidentaux s'installent au Proche-Orient avec des buts religieux et politiques auxquels s'ajoutent des objectifs commerciaux et économiques. Au XVI ${ }^{e}$ siècle, quand le latin commence à décliner pour laisser place aux langues nationales considérées comme vulgaires jusqu'alors, les capitulations signées entre Soliman le Magnifique et François 1er, en 1535, ouvriront les portes de l'Empire et du ProcheOrient, non pas seulement aux commerçants occidentaux, mais aussi aux missionnaires qui mettent en place des écoles au sein des monastères sous la protection de l'Église catholique (Ekrem 1934 : 403-410). C'est ainsi que les Français acquièrent des privilèges intéressants dans le domaine commercial surtout, mais aussi politique et militaire, mais ces derniers resteront secondaires. Commencent ainsi l'installation des commerçants sur le territoire de l'Empire ottoman et par la suite l'ouverture des écoles francophones. Le mouvement prendra de l'ampleur avec toutes les conséquences qui en découlent quand, à l'époque de Henri IV, en 1604, avec l'acte de la cinquième capitulation, ces privilèges seront reconnus à d'autres chrétiens des pays dont les rois sont alliés ou amis du roi de France, ou à ceux qui voyagent sous la protection du drapeau français (Ekrem $1934:$ 420-425).

Dès 1583, sur la demande des Latins du quartier Galata, et par l'intermédiaire de l'ambassadeur de France cinq pères jésuites étaient venus s'installer à l'église SaintBenoît à Istanbul et fonder la première école étrangère chez les Ottomans. C'est dans cette école qu'ont été formés en langue turque et arabe les enfants des familles 
vénitiennes habitant à Istanbul ou venant de Venise, appelés 'Giovanni della lingua' (Polvan1952 : 98-99, 145-150).

3 En 1669 fut fondée, par Colbert, ministre de Louis XIV, dans le couvent capucin du Palais de France à Istanbul, l'école des Enfants de langue et Drogmans ${ }^{1}$ qui, malgré la création en 1795, par le gouvernement révolutionnaire, de l'École des langues orientales de Paris, continua à former également les jeunes Français au métier d'interprète jusqu'à sa destruction dans un incendie en 1831. Ces jeunes qui avaient entre 17 et 19 ans, venaient, au départ, de France pour la plupart, et par la suite, des familles commerçantes installées au Levant. Au bout de 3 à 5 ans d'étude du turc, de l'arabe et $d u$ persan ils devenaient traducteurs et travaillaient au service de l'Ambassade de France à Istanbul ou dans les consulats français des comptoirs de l'Empire comme Smyrne, Alep, Tripoli, Saida, Chypre, Eubée, Thessalonique, Morée, etc. au point que le nombre des traducteurs atteignait en 1730,52, à raison de 13 à Istanbul et 39 en province (Uzunçarsili 1984 : 314). La délicate mission des drogmans était de rendre les subtilités des propos diplomatiques échangés pendant les cérémonies au Sérail impérial et d'entretenir des relations de travail avec les fonctionnaires de la Sublime Porle ${ }^{2}$... Beaucoup de diplômés dont Antoine Galland, devenant de véritables érudits et spécialistes de ces langues ont fait connaître à l'Occident les civilisations orientales en traduisant le Coran, les Fables de Nasreddin Hodja, les Mille et une Nuits...

Bref, au XVII ${ }^{e}$ et dans la première moitié du XVIII ${ }^{e}$, l'enseignement du français est assuré par les missionnaires qui donnent les connaissances de base de leur religion aux chrétiens vivant sur le territoire ottoman, et forment les interprètes et traducteurs pour les ambassades et consulat ${ }^{3}$.

\section{Le système éducatif}

5 L'éducation est assurée par l'État. Comme il s'agit d'un État basé sur l'Islam et que le sultan est également khalife, la politique éducative est organisée selon les attentes du peuple musulman, mais les minorités ont droit à des privilèges éducatifs. L'enseignement primaire est assuré dans les mektep/écoleset l'enseignement supérieur dans les medrese/madrasa-el-azhar qu'on ne trouve que dans les grandes villes. Il n'existe pas d'enseignement secondaire au sens actuel.

6 Dans les mektep, l'apprentissage commence par l'alphabet arabe, ensuite vient la lecture du Coran, une lecture sans aucun souci de sens, jusqu'à un apprentissage par cœur. Les cours de religion, de lecture, d'écriture ne préparent pas l'enfant à la vie, mais sont censés assurer le salut éternel... En ce qui concerne les medresè, l'enseignement toujours en arabe et coupé du monde réel, des faits de la nature et de la société est basé sur le rabâchage des règles de grammaire, la mémorisation de textes, le spiritualisme, la contemplation etc. Les diplômés devenaient imam, instituteur, et surtout kadi, ou montaient, en occupant différents postes au sein de l'établissement, dans la hiérarchie, laquelle ne différait pas beaucoup du clergé chrétien, pour devenir müderris/professeur (Karal $1988: 5-6)$.

7 Peu à peu le mot mektep s'identifiera aux écoles publiques créées par l'État sur le modèle occidental et s'opposera ainsi aux medresè qui, considérés comme des organisations religieuses traditionnelles, ne furent l'objet d'aucun effort de 
modernisation jusqu'à l'époque de la IIe monarchie, autrement dit la Révolution de 1908.

\section{Les premières tentatives de modernisation : réformes militaires}

Fondé en 1299, l'Empire ottoman connut son apogée au seizième siècle après la prise de Constantinople en 1453, suivie d'une rapide expansion en Europe continentale et en Méditerranée. Mais au début du XVIII ${ }^{\mathrm{e}}$ siècle, le déclin avait déjà commencé pour cet Empire qui inspirait quelques décennies avant Racine (Bajazet), Molière (Le Bourgeois gentilhomme), Mlle de Scudéry (Le Grand Cyrus), etc. En effet avec le Traité de Karlowitz en 1699 il ne perdait pas seulement la Hongrie, la Transylvanie, la Podolie, l'Ukraine, la Morée, Corinthe et Azov, mais aussi les tributs qu'assuraient ces pays chrétiens. Il ne s'agit donc plus ni d'une politique d'expansion à l'extérieur, ni d'une puissance du sultan à l'intérieur. C'est dans la quête de cette force révolue que va commencer le mouvement de la modernisation. Le sultan Ahmet III (1703-1730) avec Rocheford, Mahmut 1er (1730-1754) avec le comte de Bonneval entreprennent des rénovations dans le domaine militaire qui vont durer sous les règnes de Mustafa III (1757-1774) avec le baron de Tott et Abdülhamit 1er (1774-1789) avec Aubert, Le Roi et Durest (Bois 1993 : 152-160). C'est à la même époque que par l'intermédiaire de l'ambassadeur de la Sublime Porte en France l'imprimerie est introduite dans l'empire avec trois siècles de retard sur l'Occident ${ }^{4} \ldots$ Les rénovations se poursuivent avec le sultan Selim III (1789-1807) dont le règne s'identifie avec Nizam-i Cedit autrement dit le Nouvel Ordre qui, dans un sens large, signifie toutes les entreprises de ce dernier pour réformer l'armée et surtout le corps des janissaires, pour supprimer les privilèges de la classe des ulema (savants) et pour améliorer, sur le modèle européen, les sciences, les arts, l'agriculture, le commerce. C'est à cette époque que pour la première fois l'Empire ottoman établira des ambassades en Europe : à Vienne, à Berlin, à Paris et à Londres, ce qui signifie changement d'une mentalité qui méprisait l'Europe jusqu'alors (Karal 1988 : 60-73). Rappelons en passant que ces entreprises, et surtout la réforme de l'armée, provoquent au sein des janissaires un mécontentement qui lui est fatal (1807, la Révolte de Kabakçi).

\section{La rénovation du système éducatif : l'époque des écoles (1773-1839)}

9 On a tendance à prolonger l'époque des écoles jusqu'à l'année 1923, autrement dit jusqu'à la destruction de l'Empire ottoman et la proclamation de la République turque. En effet après l'Edit de Tanzimat (1839) les réformes se succèdent aussi bien dans le domaine de l'éducation que dans les structures de la société. Mais les premiers mouvements de modernisation entrepris par l'État : la création des écoles militaires dès 1773, la mise en place d'un cours d'interprétation à Bâb-i Ali (1821), le firman de Mahmut II pour l'éducation de base obligatoire (1824), la suppression du corps des janissaires (1826), la parution du premier journal en turc (Takvim-i Vekayi, 1831) datent de cette période qui s'étend de 1773 à 1839 . 
10 En Europe, le XVIII ${ }^{e}$ siècle est le siècle où la France s'affirme comme le pays le plus puissant où le français devient la langue de la diplomatie internationale. Le Traité de Küçük Kaynarca signé le 21 juillet 1774 entre l'Empire russe et la Sublime Porte est le premier texte rédigé en français. L'Empire ottoman qui avait commencé par moderniser et transformer l'artillerie, la marine et l'armement en faisant venir des ingénieurs et des conseillers militaires, français pour la plupart, se rend compte de la nécessité d'une rénovation dans le domaine éducatif... Parmi les différents établissements créés à cette époque, trois écoles militaires sont importantes du fait de la présence du français : la première est Riyaziye Mektebi (l'École des sciences mathématiques), rénovée en 1776 elle est devenue Hendesehane-i Bahrî-i Hiimayun et a été réouverte définitivement en 1783 sous le nom de Mühendishane-i Bahrî-i Hümayun (École navale militaire/impériale) sous le règne de Abdülhamit 1er (1774-1789). Selim III (1789-1807) continue dans la même voie en créant, vingt ans après, en 1793 une autre école intitulée Mühendishane-i Berrî-i Hümayun (École militaire/impériale d'artillerie) pour l'artillerie cette fois-ci et qui a été réorganisée en 1795 (Berkes 1978 : 93). Le mouvement prendra de l'ampleur par la suite, notamment avec Tiphane-i Amire ve Cerrahane-i Mâmure, la Faculté de médecine.

\section{Mühendishane-i Bahrî-i Hiimâyûn-1773 (École navale militaire/impériale)}

11 Après la destruction de la flotte ottomane en 1770 à Çeçme, la modernisation de la flotte était apparue nécessaire dans un empire animé par une logique de conquête. En 1773, vers la fin du règne de Mustafa III $^{5}$, vu les besoins techniques, une école navale verra le jour marquée par la présence des spécialistes et des professeurs français qui donneront pour la première fois des cours de français dans une institution impériale. Un autre progrès introduit par cette école est l'absence de cours de religion et une utilisation systématique du matériel. Au début, comme il n'existait pas d'école qui fournirait des élèves, on recruta des enfants qui ne savaient ni lire, ni écrire. Il ne faut pas oublier que, jusqu'à la fin du XVIII ${ }^{e}$ siècle, il était défendu aux musulmans d'apprendre une langue occidentale. A la fin du XVI ${ }^{e}$ siècle, même les mathématiques et la géométrie avaient été supprimées des programmes des medresè, sous prétexte que ces matières étaient incompatibles avec la religion.

Les programmes étaient de niveau primaire et partiellement secondaire. On commençait par l'enseignement de la lecture et de l'écriture, ensuite on introduisait l'arabe, le persan et le français, et finalement venait l'enseignement des mathématiques et des sciences maritimes. Le premier enseignant était un marin confirmé appelé Seyit Hasan l'Algérien qui maîtrisait bien les langues occidentales.

Plus tard on recruta les enfants des capitaines et des officiers, qui avaient appris à lire le Coran et connaissaient l'écriture arabe. On introduisit dans les cursus qui étaient de trois ans. en première et en deuxième année qui constituaient des troncs communs, des cours comme le calcul, l'arabe, les mathématiques, la géométrie, l'algèbre, le dessin. En troisième année commençait la spécialisation et l'étude des disciplines concernées.

A partir de 1842 le français devient facultatif et l'anglais obligatoire (Akyüz 1993 : 125). 


\section{Mühendishane-i Berrî-i Humayun-1793 \\ (École Militaire / impériale d'artillerie)}

15 Créée vingt ans après l'École navale par Selim III et réorganisée en 1795, cette école d'artillerie militaire avait pour objectif, d'une part, de diffuser des sciences comme la géométrie, les mathématiques, la philosophie, la morale, la physique, la chimie, la géographie et d'enseigner de toute urgence l'industrie de guerre dont le Sublime Empire avait besoin. Il faut ajouter que les méthodes et les techniques d'enseignement de ces deux écoles militaires seront unifiées dès la création de la seconde.

Le cursus était de 4 ans. On enseignait, les deux premières années, l'arabe et le français. Le mardi et le vendredi étaient fériés, le lundi et le jeudi étaient consacrés aux travaux pratiques sur le terrain. La création d'une telle école et le recrutement de professeurs assez prestigieux qui constituent un progrès considérable attirent l'attention des pays européens qui la soutiennent par l'offre et l'envoi de manuels, notamment la France de Napoléon qui voulaient gagner l'amitié du sultan. On trouve dans l'école du matériel de mesure en bronze pour les cours de géométrie (Akyüz 1993 : 126). La bibliothèque comptait 400 livres en français pour la plupart, sur l'art militaire, la physique, l'artillerie, les mathématiques, l'armement et la fameuse Encyclopédie française (Karal $1988: 68)$.

17 Et pour la première fois dans le système éducatif ottoman, c'est dans ces écoles militaires que les cours de français sont assurés obligatoirement par un Français (Karal 1988 : 194), accompagné par un répétiteur. Dans la même école, sous la direction du professeur Abdurrahman Çelebi, sera créée la seconde imprimerie turque (soixante-six ans après la première) où de 1795 à 1802 seront imprimés cinq manuels pour Muhendishane (Baysal 1968 : 61-62). Le premier livre en français écrit par un Turc pour enseigner le turc aux étrangers fut également imprimé à la même époque dans cette imprimerie (Tanpinar $1955: 16$ ).

\section{Tiphâne-i Amire ve Cerrahâne-i Mâmure-1827 (L'école de médecine et l'école de chirurgie)}

Dès 1805 , une autorisation fut accordée aux Grecs de créer une école de médecine qui fut fermée en 1812. Une autre tentative échoua également en quelques mois en 1807.

C'est finalement sous le règne de Mahmut II, en 1827, que fut créée la première école de médecine qui est suivie par une école de chinirgie un ou deux ans après. Rénovées en 1831 les deux écoles sont unifiées en 1838. Intitulée désormais Mekteb-i Tibbiye-i Aliye-i Sâhâne (École supérieure impériale de médecine) et transférée à Galatasaray en 1839 cette école subit une réforme radicale avec le recrutement du Dr. Charles Ambroise Bernard (Berkes 1978 : 180). A l'école de médecine, l'enseignement est divisé au départ en deux cycles : le premier étant une sorte de classe préparatoire, on y enseigne l'arabe, le français et la religion, et dans le deuxième cycle les mathématiques, les sciences médicales, etc. Mais la difficulté à enseigner les langues, les sciences médicales et la religion en 4 ans, nécessite une révision des programmes et oblige à prolonger la scolarité à 6 ans. L'enseignement est toujours en français. La religion et l'arabe sont supprimés. 
20 Le Sultan Mahmut II qui assiste personnellement à la cérémonie d'ouverture le 14 février 1839 explique, dans son discours, pourquoi l'enseignement est dispensé en français ${ }^{6}$ :

21 Le voyageur anglais Charles MacFarlane (1850) qui, apparemment, n'aimait pas beaucoup les matérialistes français fut scandalisé en visitant la bibliothèque, en 1847, de voir une collection qui réunissait tant de livres matérialistes. Par ailleurs, un jeune Turc était en train de lire le Système de la Nature (1770) du Baron d'Holbach, un autre Le compère Mathieu dans Jacques le fataliste de Diderot. Il voit sur les rayons de la bibliothèque les Rapports du physique et du moral de l'Homme de Cabanis qui parle de l'absurdité de croire en Dieu et de l'impossibilité de la mort de l'âme. Un jour pendant qu'il visitait l'hôpital militaire à Scutari, il remarque un livre sur un canapé dans une salle réservée aux médecins et aux assistants. C'est la dernière édition du Système de la Nature, encore une fois. En feuilletant ce livre, il remarque les notes prises en marge, ce qui est le signe d'une lecture sérieuse, au même moment un futur médecin s'approche et lui dit : "C'est un grand ouvrage ! C'est un grand philosophe. Il a toujours raison ". MacFarlane constate, d'autre part, que les étudiants travaillent tout seuls sur des cadavres, s'approche d'eux pour leur demander si ce n'est pas un sacrilège d'après la religion musulmane. Et un étudiant de lui répondre en français: « Eh ! Monsieur ce n'est pas au Galataseraï qu' il faut venir chercher la religion! (Berkes 1978 : 228-229).

$\mathrm{Au}$ début du XIX ${ }^{\mathrm{e}}$ siècle, une autre tentative importante de modernisation de l'empire arrive avec l'autorisation accordée par Mahmut II aux musulmans d'être nommés interprètes et traducteurs. En effet, la dégradation de la situation de l'Empire, le fait que le métier d'interprète devienne presque le monopole des Grecs et que ces derniers soient en bons termes avec les missions étrangères, avaient poussé le sultan à mettre en place des cours de français au sein de la Sublime Porte. Ces cours deviendront en 1821 la Chambre d'Interprétation qui se transformera en 10 ans, en une véritable école contribuant non seulement à la formation des interprètes, mais aussi à celle des diplomates et des intellectuels du pays.

La promulgation en 1839 de l'Edit de Tanzimat (Hat-ti Chérif ou Charte des Turcs) ouvre une nouvelle ère de libéralisation et de modernisation. C'est grâce à cette chambre d'Interprétation qu'on traduira en Turc les œuvres des plus grands auteurs et philosophes, en particulier Emile, Tartuffe, une anthologie de dialogues intitulée Mahaverat-i Hikemiye extraits de Fénelon, Fontenelle et Voltaire par Miinif Pasa, Tercüme-i Manzume par Sinasi et Telemak Tercümesi par Yusuf Kamil Pasa qui, en 1283 (1866), a déjà été publié quatre fois. D’après Baysal (1968 : 14,54) de 1729 à 1830, autrement dit en un siècle, on compte quelque 180 livres imprimés, tandis que de 1830 jusqu'à la monarchie constitutionnelle (1876) le nombre de livres imprimés atteint 3066. Et sur les quelque 185 traductions publiées entre 1729 et 1875, 109 (soit 54 \%) sont traduits du français.

\section{Pour conclure}

Jusqu'à la monarchie constitutionnelle, le français est la seule langue enseignée dans les établissements publics. Sauf dans les établissements privés des minorités, il gardera sa suprématie et sa place de première langue jusqu'aux années $50 \mathrm{du} \mathrm{XX}^{\mathrm{e}}$ siècle où les États-Unis commencent à s'affirmer comme l'une des plus grandes puissances du monde et proposent, avec le plan Marshall, une aide financière aux pays d'Europe sortis 
épuisés de la Seconde Guerre mondiale. C'est ainsi que l'anglais commence à bousculer le français qui devra céder sa place à l'allemand à partir des années 60 quand la migration de la main d'œuvre turque en Allemagne Fédérale favorisera l'introduction de cette langue en Turquie.

Aujourd'hui la décision prise par les institutions européennes d'introduire une seconde langue étrangère dans les programmes contribue largement à un maintien du français comme la seconde langue étrangère et les retombées sur les jeunes générations de l'apprentissage de cette langue et de cette culture continuent d'étonner d'autres voyageurs contemporains...

\section{NOTES}

1. Le mot tercüman, d'origine syriaque est devenu dragomanno en italien et drogman ou truchement en français.

2. La Sublime Porte (Bâb-i Ali) au sens large fait référence à un groupe d'administrateurs constitués du grand-vizir (Premier Ministre) et de ses adjoints à savoir : Re'isü'l Küttab (ministre des Affaires extérieures), Kâhya Bey (ministre des Affaires intérieures) et des conseillers d'Etat qui, à partir du règne de Murât III (1575-1595), s'occupent effectivement de l'administration de l'État. Les conseils des bureaux qu'ils dirigeaient, au nombre de 24 au début du XIX ${ }^{\mathrm{e}}$ se tenaient dans une enceinte du Palais de Topkapi, d'où l'appellation de Sublime Porte (Mardin 1998 : 158).

3. "Ces missionaires chrétiens qui avaient reçu une formation spécifique et qui étaient bien équipés ont, à l'ombre de leurs missions spirituelles, une mission temporelle qui est de servir les intérêts économiques, culturels et politiques de leurs pays » (Akyiiz 1993 : 89).

4. Yirmisekiz Çelebi Mehmet, l'ambassadeur de la Sublime Porte à Paris et son fils Sait Efendi, qui fut par la suite le premier vizir turc à apprendre le français, jouèrent un grand rôle dans l'introduction de l'imprimerie dans l'Empire. Mais c'est un transylvanien né à Cluj, naturalisé turc et converti à l'islam, Ibrahim Müteferrika qui est traditionnellement considéré comme le fondateur de l'imprimerie. Le firman autorisant la création de l'imprimerie date de 1139 de l'Hégire (1726). D'après Baysal (1968), les Ottomans connaissaient depuis 1494 l'imprimerie. D'après Ersoy (1959 : 51-53) jusqu'à 1729 le nombre des imprimeries que possédaient les minorités et les missions étrangères était de 37. D'après Bodrumlu (1928 : 147) il était défendu aux juifs d'imprimer des œuvres en turc et en arabe.

5. Mustafa III (1757-1774) qui aimait la science et la rénovation avait un faible pour l'astrologie. Pensant que Frédéric II devait ses succès à ses bons astrologues, il lui avait demandé par l'intermédiaire de son ambassadeur, de lui envoyer trois de ses meilleurs astrologues qui lui diraient l'avenir. Alors le roi lui expliqua ses trois secrets : 1- Lire l'Histoire et en tirer des leçons, 2- Avoir une bonne armée et la former en temps de paix comme si elle allait guerroyer tout de suite, 3- Tenir le trésor toujours plein (Lavisse-Rambaud 1896 : 485-487).

6. La date et le contenu de ce discours ont été sujets à plusieurs polémiques. On peut en trouver une discussion intéressante dans la Revue Tarili ve Tapinili 83 (1990 : 38-43). 


\section{RÉSUMÉS}

$\mathrm{Au}$ XVIIIe siècle l'Empire ottoman, qui se rend compte qu'avec les anciennes techniques et méthodes il ne pourra pas l'emporter sur les pays occidentaux, commence à faire venir des ingénieurs et des conseillers militaires, français pour la plupart, en vue de moderniser et de transformer l'artillerie, la marine et l'armement. Le mouvement prendra de l'ampleur par la suite notamment avec la Faculté de Médecine.

In the 18th century, as the Ottoman Empire realized that it could not acheive victory over the Western countries with old methods or techniques, it started to invite French engineers and counselors in order to modernize the army, the navy and the arms as well. This movement then grew especially with the Faculty of Medicine.

INDEX

Mots-clés : Empire ottoman, XVIIIe siècle, système éducatif, réformes, Ottoman Empire, 18th century, reforms

\section{AUTEUR}

HANIFE GÜVEN

Université de Dokuz Eylül (TURQUIE) 